\title{
Comedication and Treatment Length in Users of Acetylcholinesterase Inhibitors
}

\author{
Anne Sverdrup Efjestad ${ }^{a} \quad$ Hege Ihle-Hansen $^{b} \quad$ Vidar Hjellvik $^{c}$ \\ Hege Salvesen Blix ${ }^{c}$ \\ a Baerum Hospital Pharmacy, Hospital Pharmacy Enterprises, South Eastern Norway, Gjettum, \\ Norway; ${ }^{b}$ Vestre Viken, HF, Baerum Hospital, Gjettum, Norway; ${ }^{c}$ Norwegian Institute of Public \\ Health, Oslo, Norway
}

\section{Keywords}

Acetylcholinesterase inhibitors - Alzheimer disease and medical treatment .

Pharmacoepidemiology of dementia - Pharmacotherapy for behavioral and psychological

symptoms of dementia

\section{Abstract}

Background/Aims: Reduced clinical effect on cognitive decline in dementia by acetylcholinesterase inhibitors (AChEIs) may be due to concurrent use of drugs with anticholinergic properties. The aim was to analyze the incidence of AChEI use and comedication with drugs with anticholinergic properties and other potential unfavorable effects. Methods: A prospective study applying drug use data from the Norwegian Prescription Database. Anticholinergic Drug Scale (ADS) scores were used as a measure of overall anticholinergic burden. Results: Patients with high ADS scores were more frequently discontinuing treatment early. Coprescribing of antipsychotics was strongly associated with early discontinuation of AChEI treatment. Conclusion: Coprescribing with potentially unfavorable medications was common. A high ADS score was associated with early discontinuation of treatment.

(C) 2017 The Author(s)

Published by S. Karger AG, Basel

\section{Introduction}

Reduced cognition and change in behavior in Alzheimer disease (AD) may be a direct result of the loss of acetylcholine [1]. Thus, to stimulate cholinergic transmission, acetylcholine esterase inhibitors (AChEIs) are used in patients with AD. About 70,000 people 
suffered from dementia in Norway in 2010 [2], worldwide 47.5 million people (2015), and there are 7.7 million new cases every year [3].

Donepezil, rivastigmine, and galantamine are currently available in Norway for the symptomatic treatment of mild to moderately severe AD. Although the clinical effect of AChEIs on cognitive function is generally limited, the individual variability in response is extensive. This variability has to some extent been explained by the prescriber, which could reflect prescription of drugs which are contraindicated in combination with AChEIs, or by individual patient variability [4]. Moreover, discontinuation of AChEIs or switch to another AChEI can be related to environment (hospitalizations/change of care), medications (adverse drug reactions to AChEIs and inappropriate concurrent use of anticholinergic agents), and patient factors (cognitive decline, anxiety, and weight loss) [5-8]. Rapid cognitive decline has been shown to be linked to switching [8], indicating that the decision to switch to another AChEI may be made for a potentially better therapeutic response. A drug holiday may be undertaken to assess the real benefit of AChEI use. AD-related hospitalization has been shown to be the strongest predictor of discontinuation [8].

Patients with dementia are frequently treated with psychotropic drugs such as antidepressants, anxiolytics, antipsychotics, and hypnotics/sedatives for behavioral and psychological symptoms of dementia (BPSD) as part of the dementia syndrome. Management of these symptoms is difficult due to the risk of serious adverse effects and due to the absence of credible alternatives $[9,10]$. The use of psychotropic drugs may result in considerable morbidity, especially in the elderly who are more susceptible to both effects and adverse effects of drugs $[11,12]$. Polypharmacy is a well-known phenomenon in older adults, with increased risk of interactions [13].

Reduced clinical effect of AChEIs may be due to concurrent use of interacting drugs with anticholinergic properties [1, 14-16]. The prevalence of coprescribing of anticholinergic drugs in the AChEI-treated patients in Norway is unknown. Many commonly used drugs have primary or secondary anticholinergic activity, e.g., drugs for urinary frequency and incontinence (urologic spasmolytics), antihistamines, antidepressants, and antipsychotics [1, 12, 14-17]. Moreover, there is a vast number of agents expressing anticholinergic activity in vitro [18]. These could possibly interact and have a synergistic effect when administered together with other anticholinergics. Anticholinergic Drug Scale (ADS) score models have therefore been developed as assessment tools for the evaluation of the overall anticholinergic burden in patients [17-20] and comprise agents from several drug classes. Total ADS scores have been shown to be associated with cognitive dysfunction [21], resulting in low Mini-Mental State Examination scores [20,22] and increased mortality in older patients [22, 23]. Moreover, cognitive impairment has been found to be associated with polypharmacy [13] and increased mortality in the elderly [22].

The aim of the study was to analyze the incidence of prescribed AChEI treatment in Norway, to which extent incident users became persistent users ( $\geq 8$ months treatment), to which extent persistent AChEI users differed from nonpersistent AChEI users in regard to age, sex, and prescriber, and to which extent comedication (with focus on drugs with anticholinergic properties) was associated with treatment length in persistent AChEI users.

\section{Materials and Methods}

Data

Drug use data were collected from the Norwegian Prescription Database (NorPD). This database covers the total population and, since 2004, information from all prescriptions dispensed at Norwegian pharmacies to individual patients has been included in the database. 
Fig. 1. Flow chart. Time for study period, all users of acetylcholinesterase inhibitors (AChEIs), new users of AChEIs, persistent users, quitters and subgroup for concomitant treatment.

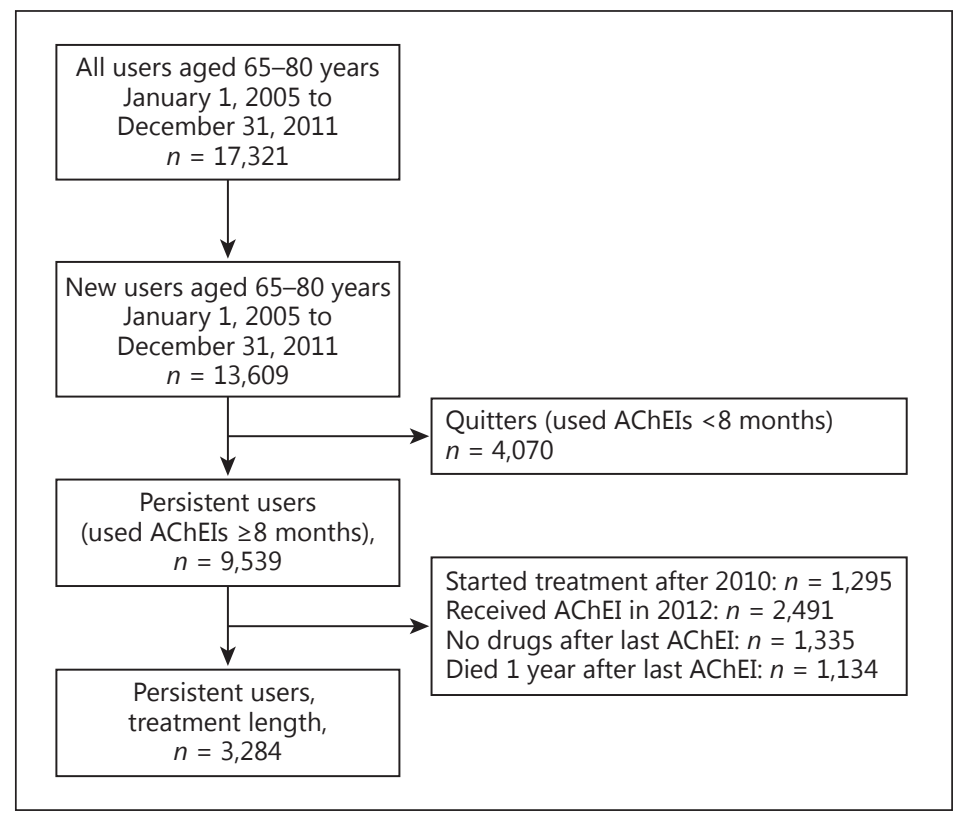

It is possible to follow all individuals who have purchased medicines through their personal unique identification number [24]. Drugs dispensed to patients in institutions are not included in the NorPD, so when a patient moves to a nursing home, we are not able to follow the patient's drug use any more.

Data registered in the NorPD include sex, age, year of death (when relevant), and information on drugs dispensed. In this study, we assumed that dispensed drugs represented consumed drugs. The drugs were classified according to the Anatomical Therapeutic Chemical classification system, version 2015 [25]. For each dispensed prescription, the following data were obtained: the date of dispensing, medicinal product name and formulation, Anatomical Therapeutic Chemical code, defined daily doses and the number of tablets/ capsules/plasters (allowing us to calculate treatment periods), and the prescriber's specialty. Specialists in general medicine or geriatric medicine are the specialists who in practice initiate antidementia treatment in Norway and were considered specialists in the care of patients with dementia; all other physicians were considered nonspecialists.

\section{Users and Incident Users of Antidementia Drugs}

Users of AChEIs registered in the NorPD in the period from 2005 to 2011 were eligible for inclusion in the study (Fig. 1). Persons using solely the antidementia drug memantine were not eligible. Incident use was defined as being prescribed an AChEI drug after $>365$ successive days with no AChEI prescriptions. Some patients were repeated incident users (having several AChEI-free periods of $>365$ days). For each patient, we started follow-up at the date initiating the longest period with subsequent prescriptions $<365$ days apart (index date). The "longest period" means the period with the largest number of prescriptions. Treatment length was estimated as the time from the index date until the drug dispensed in the last prescription of the longest period was supposed to be consumed. The earliest possible index date was January 1, 2005. To allow for at least 1 year of follow-up, we only included patients with an index date before January 1, 2012. Users that were younger than 65 years or older than 80 years at the start of follow-up were excluded. For repeated incident users, only the treatment periods starting when the individual was between 65 and 80 years 
were considered. The 80-year limit was set because older patients with dementia are likely to become nursing home residents during the follow-up period, whereupon many of the residents will stop the AChEI treatment. For nursing home residents, medication is not registered in the NorPD. The lower limit of 65 years was chosen due to the definition of "elderly" [26].

\section{Persistent Users and Quitters}

New guidelines for dementia treatment are under revision in Norway, but currently, both specialists in geriatric medicine and general medicine can initiate and evaluate clinical effects of AChEI treatment. Potential response to treatment should be evaluated 3-6 months after initiation including measures of cognitive, global, functional or behavioral symptoms. Furthermore, possible adverse drug effects should be assessed. We chose 8 months (240 days) as a cutoff to leave a lag time for physician evaluation visits. The patients being prescribed AChEI treatment for $<8$ months are called quitters, the remaining are referred to as persistent users.

A user was defined as persistent if any of the following was true: (a) a new prescription was given between day 210 and day 240 after initiation (index date), (b) drugs for at least 210 days' consumption were prescribed during the first 210 days from initiation, or (c) the last prescription before day 210 lasted to day 210. A stricter definition (either drug for at least 240 days prescribed during the first 240 days or the last prescription before day 240 lasting to day 240) resulted in fewer persistent users. However, $29 \%$ of those who were defined as nonpersistent by the strict definition were prescribed AChEI drugs after the 240-day limit ("false quitters"), compared to $16 \%$ using the first definition; thus, the first definition was chosen to reduce the number of "false quitters."

\section{Treatment, Coprescribing and Anticholinergic Scores}

To investigate the association between coprescribing and treatment length, we defined a subgroup of persistent users for whom we could assume that we actually knew the treatment length, and for whom the end of treatment was not due to conditions leading to death shortly thereafter. It is reasonable to assume that many patients will be institutionalized during follow-up. However, for these patients, we cannot know whether they continue using AChEIs in the institution since drugs distributed in institutions are not registered in the NorPD. Since the majority of AChEI users also used other drugs, disappearance from the NorPD altogether would be a strong indication of institutionalization (or emigration) rather than the end of AChEI treatment. For this analysis, we therefore excluded users who: (1) were not registered in the NorPD after the last AChEI prescription (probably institutionalized or emigrated), (2) had the last prescription in 2012 (might have continued after 2012), (3) started treatment in 2011 (short follow-up) or (4) died the same year or the year after the last prescription (probably nondeliberate end of treatment). For this group, coprescribing was defined as being prescribed another medication (see selected drug groups below) during the first 6 months after initiation. AChEI users with different treatment duration were compared, and the 6-month coprescription window was chosen to make the length of the coprescription window independent of treatment duration. The 6-month period for comedication was chosen independently of the 8-month cutoff limit used to define persistent users.

We registered coprescribed drugs with anticholinergic properties. Urologic spasmolytics (G04BD) have substances with anticholinergic properties and may be initiated due to incontinence prevalent in patients with dementia. However, incontinence is also a common adverse effect of AChEIs [15]. Dementia in Parkinson disease is common and rivastigmine is licensed for cognitive impairment in Parkinson disease, hence antiparkinson drugs (N04) were selected. Anxiolytics (N05B), hypnotics/sedatives (N05C), antipsychotics (N05A), antide- 
Sverdrup Efjestad et al.: Comedication and Treatment Length in Users of

Acetylcholinesterase Inhibitors

pressants (N06A), and analgesics (N02) are frequently used in older age, but have anticholinergic activities or other known adverse effects in patients with dementia.

To compare the prevalence of the drugs listed above in AChEI users with the prevalence in the general population, we computed the age-adjusted 6-month prevalence ratio $\left(\mathrm{PR}_{\mathrm{A}}\right)$ by dividing the prevalence in the AChEI users by the age-adjusted prevalence in all 65- to 80-yearolds in Norway (including the AChEI users) in the 6-month period July to December 2007, with the AChEI users as standard population.

The ADS score for each AChEI user was calculated. The basis for the ADS score evaluation is a list of drugs and their anticholinergic scores [27] founded on previously published classifications and studies $[16,18,19,28]$. The ADS score is from 0 to 3 , where 0 is defined as "no anticholinergic activity," 1 as "potentially anticholinergic," 2 as "clinically significantly anticholinergic," and 3 as "strong anticholinergic activity." The ADS score for each AChEI user was calculated as the sum of the ADS scores for each different drug prescribed during the 6-month coprescription window.

\section{Statistical Analysis}

$\mathrm{R}$ version 3.1.0 [29] was applied for descriptive statistics; period prevalence, means, medians, percentages and 95\% confidence intervals (CIs) are presented when appropriate. The R-function prop.trend.test was used to test for associations between comedication at the start of treatment and treatment length. Treatment periods were calculated based on number of tablets/capsules and dose intervals.

\section{Ethics}

The NorPD generated anonymous files for research purposes, as regulated by Norwegian law for health registers [24], hence there was no demand of additional approval by the ethics committee.

\section{Results}

17,321 persons aged 65-80 years were registered users of AChEIs in the 7-year period from January 1, 2005 throughout 2011, and 13,609 of these were incident users; 13,056 (96\%) initiated treatment once, while $534(3.9 \%)$ and $19(0.1 \%)$ started treatment 2 and 3 times during the period, respectively, i.e., they had 1 or 2 intervals of at least 1-year duration without any AChEI prescriptions. For 324 patients (59\%) of those with $>1$ treatment period, the second or third treatment period was the longest.

The mean age of the 13,609 incident users at first prescription was 75.1 years (interquartile range 73-78), and 7,961 (58.5\%) were women. The most frequently used AChEI was donepezil, followed by rivastigmine and galantamine. The prescription patterns have changed over the years; i.e., annual incidence rates have decreased for donepezil and galantamine and increased for rivastigmine. Moreover, the use of memantine has increased, and combined use of AChEIs and memantine increased from $4.2 \%$ in 2004 to $8.6 \%$ in 2012 in prevalent users of all ages.

Around one-third of the incident users $(4,070)$ were prescribed AChEIs for $<8$ months and were considered to be quitters (Fig. 1). Persistent users and quitters did not differ in age and gender, but a higher proportion of persistent users had their treatment initiated by a specialist in geriatrics (16.4\% [95\% CI 15.7-17.2] vs. 14.2\% [13.2-15.3]).

Unfavorable Drug Combinations - Concomitant Coprescription

In the subgroup of persistent AChEI users with known treatment length $(3,284$ persons) (Fig. 1), a high proportion of coprescription was observed, being the highest for sedatives/ 


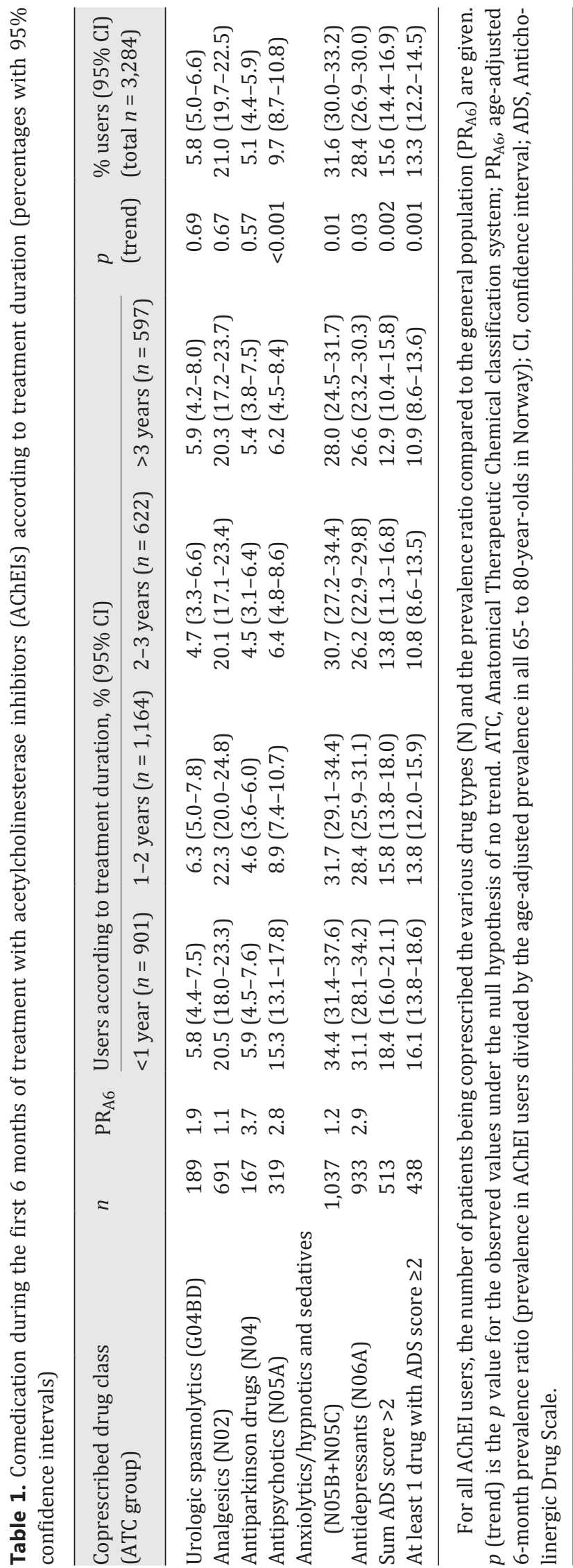


Sverdrup Efjestad et al.: Comedication and Treatment Length in Users of

Acetylcholinesterase Inhibitors

hypnotics (31.6\%) and antidepressants (28.4\%) (Table 1). The most frequently used antidepressant (N06A) was escitalopram (taken by $41 \%$ of those who used antidepressants), followed by mirtazapine (16\%), citalopram (14\%), and mianserin (14\%). Only 8\% of the antidepressant users in the study population were administered a tricyclic antidepressant (N06AA, nonselective monoamine reuptake inhibitors, with anticholinergic effect). Analgesics and antipsychotics were used by 21 and 10\%, and antiparkinson drugs and urologic spasmolytics by 5 and $6 \%$, respectively. Concerning overall anticholinergic burden, $13.3 \%$ of the patients ( 438 users) had at least 1 drug with an ADS score $\geq 2$, and 15.6\% (513 users) had a total ADS score $\geq 2$.

Compared to the general population, the age-adjusted prevalence in this population was about 4 times as high for antiparkinson drugs $\left(\mathrm{PR}_{\mathrm{A} 6}=3.7\right), 3$ times as high for antidepressants and antipsychotics $\left(\mathrm{PR}_{\mathrm{A} 6}=2.9\right.$ and 2.8, respectively), and twice as high for urologic spasmolytics $\left(\mathrm{PR}_{\mathrm{A} 6}=1.9\right)$. For anxiolytics, hypnotics/sedatives and analgesics, the prevalence was similar in the study population as for the general population $\left(\mathrm{PR}_{\mathrm{A} 6}=1.2,1.2\right.$, and 1.1 , respectively).

There was a strong association between comedication with antipsychotics during the first 6 months of treatment and duration of treatment (Table 1). While $15.3 \%$ of those with a treatment length $<1$ year were comedicated with antipsychotics, only $6.2 \%$ of those with $>3$ years of treatment were. A significantly higher proportion of those with $<1$ year of treatment than of those with $>3$ years of treatment had at least 1 drug with an ADS score $\geq 2$ (16.1 vs. $10.9 \%$ ) and a total ADS score $\geq 2$ (18.4 vs. $12.9 \%$ ). For antidepressants and anxiolytics/ hypnotics and sedatives, there was also a significant trend towards shorter treatment with a higher degree of comedication, whereas for urologic spasmolytics, analgesics, and antiparkinson drugs, treatment length was not associated with comedication during the first 6 months.

The persistent users with $<1$ year of treatment were comparable to the 4,070 quitters with regard to comedication. More quitters used antiparkinson drugs (8.6\% [7.8-9.5\%] vs. $5.9 \%$ [4.5-7.6\%]) and fewer quitters used antidepressants $(26.7 \%$ [25.3-28.0\%] vs. 31.1\% [28.1-34.2\%]). For the other drug classes and the ADS scores in Table 1, the confidence intervals overlapped.

\section{Discussion}

17,321 users of AChEIs were registered in the period, including 13,609 new users. Among those with known treatment length, the highest proportions of comedication were observed for sedatives/hypnotics and antidepressants. There was a strong association between comedication with antipsychotics during the first 6 months of treatment and duration of treatment. Patients with a high ADS score more frequently discontinued treatment than those with a lower ADS score. Concerning overall anticholinergic burden, most of the anticholinergic drugs used were considered to have a clinically significant or strong anticholinergic effect.

The incidence of dementia in Norway is around 10,000 persons every year, including 6,000 with suspected AD [30]. The number of incident AChEI users over the 7-year period was 13,609 persons, indicating that drug treatment for slowing down decline in cognition is uncommon. This may be due to late diagnosis, a high proportion of mixed dementia or low confidence in the therapeutic effect. Pharmacoepidemiological studies have reported that $10-50 \%$ of patients treated with AChEIs are prescribed anticholinergic agents [14-17]; in our study, the result was about $15 \%$. The discrepancy between the studies may be due to different definitions of anticholinergic drugs, as well as different study populations and prescription patterns. 
Improved clinical outcome may be expected with reduced anticholinergic burden in prescribed drugs. The highest proportions of comedication were observed for anxiolytics, hypnotics/sedatives, and antidepressants. This is consistent with other studies reporting that pharmacological approaches are often required for treatment of BPSD [31]. BPSD and insomnia may be part of the dementia syndrome, and insomnia is also an adverse effect of AChEIs. However, the use of anxiolytics, hypnotics/sedatives, and analgesics was similar in the study population as for the general population. Pain-related diagnosis has been shown to be associated with higher use of psychotropics in persons with dementia [32]. Pain-related conditions might be inappropriately treated with psychotropics, and more specific and better treatment of pain is thought to have a positive effect on mood [33].

The high proportion of comedication with antidepressants (N06A) indicates that depression is a major symptom in the dementia syndrome, and that many of the antidepressants are well tolerated. Selective serotonin reuptake inhibitors are recommended in several guidelines for comorbid depression in patients with AD [9], although evidence is limited [31]. Escitalopram and citalopram have shown superiority over placebo for reducing agitation in patients with $\mathrm{AD}$, but the combination may have additive negative effects since both escitalopram/citalopram and AChEIs may result in a prolonged QT interval (time between the start of the $Q$ wave and the end of the $T$ wave in the heart's electrical cycle) [34]. Nausea and diarrhea are common adverse effects of both serotonin reuptake inhibitors and AChEIs and can contribute to discomfort and weight loss [35].

Urologic spasmolytics are often used to treat incontinence, which could be caused by both the dementia syndrome and an adverse effect of AChEIs $[14,15]$. Notably, the ADS scores of urologic spasmolytic agents range from 1 (darifenacin) to 3 (tolterodine and fesoterodine). Darifenacin, a P-glycoprotein substrate with a low central anticholinergic effect, has the supposedly lowest risk of interaction with AChEIs. In our study, only $11 \%$ of the urologic spasmolytic users were prescribed darifenacin. However, due to drug interactions with inhibitors of P-glycoprotein and individual variability in brain permeability, urologic spasmolytics as well as other drugs with any central anticholinergic effect, regardless of the dose, are not recommended to people with dementia [28].

Long-term use of antipsychotics is frequently prescribed, but not recommended in people with dementia [36, 37]. The high consumption of antipsychotics in patients with AD observed in this study may reflect the difficulties in implementing guidelines to clinical practice and a lack of feasible alternatives. Antipsychotics are probably prescribed for BPSD symptoms in the initial treatment period. AChEI use for BPSD in AD may be beneficial [31,38], but the evidence is inconsistent [39]. Strong anticholinergic effects of antipsychotics may counteract the effect of AChEIs, and a QT interval prolongation and extrapyramidal side effects caused by pharmacodynamic interactions between AChEIs and antipsychotics may explain why so many patients using antipsychotics ended AChEI treatment after $<1$ year.

Nonmotor symptoms like dementia are rather common in patients with Parkinson disease, and AChEIs, especially rivastigmine, are recommended to slow the decline in cognition and activities of daily living. However, progression of motor symptoms with worsening of the tremor is frequently reported as side effects, and might limit the use [40]. In our study, only $5 \%$ were prescribed both AChEIs and antiparkinson drugs.

A higher proportion of persistent users than quitters had their treatment initiated by a specialist in geriatrics, which could be explained by geriatricians possibly having more focus on polypharmacy in elderly people than specialists in general medicine, resulting in a total lower ADS score.

The study has some limitations. The NorPD does not contain individual information on prescriptions made in institutions such as hospitals and nursing homes. Therefore, the total number of patients in Norway using AChEIs is higher than reported in this study. The age limit 
of 80 years was set because many of the older patients would have become nursing home residents during the follow-up period, but we will lose some patients with this limit. The NorPD does not include data on the use of over-the-counter drugs and herbal drugs. This may lead to an underestimation of weak pain killers in our study as paracetamol in small pack sizes are available as over-the-counter drugs. Around one-third of the patients discontinued treatment of AChEIs before 8 months and was not included in the study regarding comedication (Fig. 1). In addition, we did not have information regarding all dementia diagnoses, neither in clinical presentation nor etiology nor about comorbidities. Therefore, it is difficult to compare the AChEI use to the prevalence of dementia. Regarding the strong association between antipsychotic use and treatment length, we do not know whether early quitters stopped treatment because they had more mental deterioration with a higher use of antipsychotics, or because of higher anticholinergic burden causing a limited response to AChEI therapy. Purchased drugs were used as a surrogate for consumed drugs and may cause overestimation. However, adherence is expected to be good, as caregivers usually are responsible for drug intakes in patients with dementia.

In conclusion, a high proportion of comedication was observed in AChEI users, and patients being prescribed antipsychotics were more likely to stop AChEI treatment. In addition, patients with low ADS scores continued treatment for a longer period of time. Our findings indicate that continuation of AChEI therapy may be related to concomitant and inappropriate drug use. The many combination therapies observed demonstrate the need for regular monitoring of clinical response and potential side effects and interactions, especially in the initial phase of AChEI treatment. If necessary, agents with no or low ADS scores should be selected, as illustrated by the use of urologic spasmolytics. Future studies on comorbidity could give more information on combination therapies and strategies to maintain the already limited effect of the AChEIs.

\section{Acknowledgements}

We thank the Norwegian Institute of Public Health for help in obtaining data from the NorPD and the Hospital Pharmacies Enterprise, South Eastern Norway, for support. The authors have no financial or any other kind of personal conflicts with this paper. The authors have not received any funding beyond the usual salaries from our institution, Baerum Hospital Pharmacy, Baerum Hospital and the Norwegian Institute of Public Health.

\section{References}

1 Boudreau DM, Yu 0, Gray SL, Raebel MA, Johnson J, Larson EB: Concomitant use of cholinesterase inhibitors and anticholinergics: prevalence and outcomes. J Am Geriatr Soc 2011;59:2069-2076.

$\checkmark 2$ Hjellvik V, Engedal K, Handal M, Flaten TP, Langballe EM, Selmer R, Strand BH: Dementia in the national cause of death registry in Norway 1969-2010. Nor Epidemiol 2012;22:217-224.

3 WHO Dementia Fact Sheet No 362 (online). http://www.who.int/healthinfo/survey/ageingdefnolder/en/ (accessed August 25, 2016).

4 Gardette V, Lapeyre-Mestre M, Piau A, Gallini A, Cantet C, Montastruc J-L, Vellas B, Andrieu S: A 2-year prospective cohort study of antidementia drug non-persistency in mild-to-moderate Alzheimer's disease in Europe. CNS Drugs 2014;28:157-170.

5 Bohlken J, Weber S, Rapp MA, Kostev K: Continuous treatment with antidementia drugs in Germany 20032013: a retrospective database analysis. Int Psychogeriatr 2015;27:1335-1342.

-6 Peyro S-P, Martin J, Gaillard C, Garnier A, Mosquet B, Guillamo JS, Parienti JJ: Sudden discontinuation of antidementia drugs in moderate and severe Alzheimer's disease in a residency for dependent elderly people: a longitudinal descriptive pilot study. Therapie 2015;70:313-319. 
7 Taipale H, Tanskanen A, Koponen M, Tolppanen A-M, Tiihonen J, Hartikainen S: Antidementia drug use among community-dwelling individuals with Alzheimer's disease in Finland: a nationwide register-based study. Int Clin Psychopharmacol 2014;29:216-223.

-8 Gardette V, Andrieu S, Lapeyre-Mestre M, Coley N, Cantet C, Ousset P-J, Grand A, Monstatruc J-L, Vellas B: Predictive factors of discontinuation and switch of cholinesterase inhibitors in community-dwelling patients with Alzheimer's disease. CNS Drugs 2010;24:431-442.

-9 Azermai M, Petrovic M, Elseviers MM, Bourgeois J, Van Bortel LM, Vander Stichele RH: Systematic appraisal of dementia guidelines for the management of behavioural and psychological symptoms. Ageing Res Rev 2012;11:78-86.

10 Dementia: supporting people with dementia and their carers in health and social care. NICE clinical guideline 42, published November 22, 2006 (online). https://www.nice.org.uk/guidance/CG42 (accessed August 25, 2016).

11 Naples JG, Handler SM, Maher RL, Schmader KE, Hanlon JT: Geriatric pharmacotherapy and polypharmacy; in Fillit HM, Rockwood K, Young JB (eds): Brocklehurst's Textbook of Geriatric Medicine and Gerontology, ed 8. Philadelphia, Elsevier, 2010, pp 880-885.

12 Ghose K: Prescribing CNS drugs for elderly patients. Drugs Aging 1994;4:275-284.

13 Shah BM, Hajjar ER: Polypharmacy, adverse drug reactions, and geriatric syndromes. Clin Geriatr Med 2012;28:173-186.

14 Carnahan RM, Lund BC, Perry PJ, Chrischilles EA: The concurrent use of anticholinergics and cholinesterase inhibitors: rare event or common practice? J Am Geriatr Soc 2004;52:2082-2087.

15 Johnell K, Fastbom J: Concurrent use of anticholinergic drugs and cholinesterase inhibitors: register-based study of over 700,000 elderly patients. Drugs Aging 2008;25:871-877.

-16 Modi A, Weiner M, Craig BA, Sands LP, Rosenman MB, Thomas J: Concomitant use of anticholinergics with acetylcholinesterase inhibitors in medicaid recipients with dementia and residing in nursing homes. J Am Geriatr Soc 2009;57:1238-1244.

17 Roe CM, Anderson MJ, Spivack B: Use of anticholinergic medications by older adults with dementia. J Am Geriatr Soc 2002;50:836-842.

18 Chew ML, Mulsant BH, Pollock BG, Lehman M, Greenspan A, Mahmoud RA, Kirshner MA, Sorisio DA, Bies RR, Gharabawi G: Anticholinergic activity of 107 medications commonly used by older adults. J Am Geriatr Soc 2008;56:1333-1341.

19 Carnahan RM, Lund BC, Perry PJ, Pollock BG, Culp KR: The Anticholinergic Drug Scale as a measure of drugrelated anticholinergic burden: associations with serum anticholinergic activity. J Clin Pharmacol 2006;46: 1481-1486.

20 Mulsant BH, Pollock BG, Kirshner M, Shen C, Dodge H, Ganguli M: Serum anticholinergic activity in a communitybased sample of older adults: relationship with cognitive performance. Arch Gen Psychiatry 2003;60:198203.

-21 Ancelin ML, Artero S, Portet F, Dupuy AM, Touchon J, Ritchie K: Non-degenerative mild cognitive impairment in elderly people and use of anticholinergic drugs: longitudinal cohort study. BMJ 2006;332:455-459.

-22 Fox C, Richardson S, Maidment ID, Savva GM, Matthews FE, Smithard D, Coulton S, Katona C, Boustani MA, Brayne C: Anticholinergic medication use and cognitive impairment in the older population: the medical research council cognitive function and ageing study. J Am Geriatr Soc 2011;59:1477-1483.

-23 Mangoni AA, van Munster BC, Woodman RJ, de Rooij SE: Measures of anticholinergic drug exposure, serum anticholinergic activity, and all-cause postdischarge mortality in older hospitalized patients with hip fractures. Am J Geriatr Psychiatry 2013;21:785-793.

24 For-2003-10-17-1246. Regulations concerning the collection and processing of health data in the prescription database (online). http://www.lovdata.no (accessed August 25, 2016).

25 WHO Collaborating Centre for Drug Statistics Methodology. ATC Classification Index with DDDs 2016. Centre for Drug Statistics Methodology, Oslo. 2014 (online). http://www.whocc.no/atc_ddd_index/ (accessed August $25,2016)$.

26 WHO Health Statistics and Information Systems (online). http://www.who.int/healthinfo/survey/ageingdefnolder/en/ (accessed August 25, 2016).

27 Efjestad A: Identification and handling of combination therapies between cholinesterase inhibitor and anticholinergic drugs; master thesis (online). http://urn.nb.no/URN:NBN:no-31107 (accessed August 25, 2016).

28 Jakobsen SM, Kersten H, Molden E: Evaluation of brain anticholinergic activities of urinary spasmolytic drugs using a high-throughput radio receptor bioassay. J Am Geriatr Soc 2011;59:501-505.

29 R Core Team: R: A Language and Environment for Statistical Computing. Vienna, R Foundation for Statistical Computing. http://www.R-project.org/ (accessed August 25, 2016).

30 Engedal K: Diagnostic work-up and treatment of dementia. J Norw Med Assoc 2002;5:520-524.

-31 Gwendolen TB, White HK: Difficult behaviors in long-term care patients with dementia. J Am Med Dir Assoc 2007;8(3 Suppl 2):e101-e113.

-32 Haasum Y, Fastbom J, Fratiglioni L, Kåreholt I, Johnell K: Pain treatment in elderly persons with and without dementia: a population-based study of institutionalized and home-dwelling elderly. Drugs Aging 2011;28: 283-293.

33 Husebo BS, Ballard C, Fritze F, Sandvik RK, Aarsland D: Efficacy of pain treatment on mood syndrome in patients with dementia: a randomized clinical trial. Int J Geriatr Psychiatry 2014;29:828-836. 
34 Pasqualetti G, Tognini S, Calsolaro V, Polini A, Monzani F: Potential drug-drug interactions in Alzheimer patients with behavioral symptoms. Clin Interv Aging 2015;10:1457-1466.

35 Wang LY, Borisovskaya A, Maxwell AL, Pascualy M: Common psychiatric problems in cognitively impaired older patients. Clin Geriatr Med 2014;30:443-467.

36 Koponen M, Taipale H, Tanskanen A, Tolppanen A-M, Tiihonen J, Ahonen R, Hartikainen S: Long-term use of antipsychotics among community-dwelling persons with Alzheimer's disease: a nationwide register-based study. Eur Neuropsychopharmacol 2015;25:1706-1713.

37 Guthrie B, Clark SA, McCowan C: The burden of psychotropic drug prescribing in people with dementia: a population database study. Age Ageing 2010;39:637-642.

38 Fereshtehnejad S-M, Johnell K, Eriksdotter M: Anti-dementia drugs and co-medication among patients with Alzheimer's disease. Drugs Aging 2014;31:215-224.

39 Bishara D: Drugs for dementia. Medicine 2012;40:684-685.

40 Connolly BS, Lang AE: Pharmacological treatment of Parkinson disease. A review. JAMA 2014;311:16701683. 